\title{
Prophylactic cranial irradiation in non-small cell lung cancer: evidence and future development
}

\author{
Justyna Chalubinska-Fendler, Lucyna Kepka \\ Department of Radiation Oncology, Military Institute of Medicine, Warsaw, Poland \\ Contributions: (I) Conception and design: J Chalubinska-Fendler; (II) Administrative support: L Kepka; (III) Provision of study materials or patients: \\ All authors; (IV) Collection and assembly of data: J Chalubinska-Fendler; (V) Data analysis and interpretation: J Chalubinska-Fendler; (VI) \\ Manuscript writing: Both authors; (VII) Final approval of manuscript: Both authors. \\ Correspondence to: Justyna Chalubinska-Fendler. Department of Radiation Oncology, Military Institute of Medicine, Warsaw, Poland. \\ Email: jchalubinska-fendler@wim.mil.pl.
}

\begin{abstract}
In non-small cell lung cancer (NSCLC) brain metastases (BM) will affect up to 50\% of patients during whole disease period. BM themselves impact heavily not only on patient's prognosis but also are a source of symptoms aggravating quality of life. Standard (pemetrexed), and non-standard chemotherapy (temozolomide) in patients with NSCLC failed to prevent them from BM. In terms of systemic treatment there are promising results showed when durvalumab (PACIFIC study), osimertinib (FLAURA trial) or alectinib (JALEX study) was used. However, those substances are effective only in small cohort with ALK or EGFR alterations. Prophylactic cranial irradiation (PCI) as a non-specific treatment has proven to be a powerful tool in preventing BM without affecting overall survival in neither way. That has been proved in nearly all earlier and all recent studies-NVALT11/DLCRG-02, RTOG 0214 update, Li et al. The positive effect of BM incidence reduction may draw fear form PCI usage due to potential cognitive toxicity the PCI may cause. Results of recent trials show that after PCI only mild cognitive disorders (MCD) may arise. Promising results in terms of reducing MCD are shown when memantine is used or/and hippocampal avoidance techniques are implemented. HA in PCI seem to be cost effective but calculations were made on small-cell lung cancer cohorts. Still even recent studies did not clarify finally which patients could benefit from PCI or other forms of preventing BM. It seems that new trials should focus on younger, fit and nonsquamous histology patients and use the tests for mild cognitive disorders (MoCA, BHA) rather than screening tests for dementia (MMSE, HVLT, ADL). The main obstacle in performing new trials on PCI in NSCLC cohorts may be, however, patients' accrual, as a difficulty which occurred during latest trials.
\end{abstract}

Keywords: Cranial irradiation; prophylaxis; carcinoma; non-small cell lung cancer (NSCLC)

Submitted Oct 28, 2019. Accepted for publication Nov 08, 2019.

doi: $10.21037 /$ jtd.2019.11.36

View this article at: http://dx.doi.org/10.21037/jtd.2019.11.36

\section{Brain metastases (BM) in patients with non- small cell lung cancer (NSCLC)}

Despite the gradual treatment improvements of primary sites of NSCLC still one of the biggest challenges is how to prevent or treat BM successfully in this group. In about $15 \%$ of patients with NSCLC, the brain will be a site of first recurrence (1). At diagnosis 10-20\% of patients with NSCLC present BM. In stage I approximately $30 \%$ of patients will develop BM during the initial 2 years since diagnosis with the figure reaching $40-50 \%$ for the whole duration of treatment irrespectively of surgery or diagnostic tools used.

The current standard for diagnosing BM is MRI, which is recommended for patients with stage II or higher (and optionally used in $\mathrm{Ib}$ ) according to NCCN guidelines v7.2019 (2). Should MRI be impossible, CT with contrast is suggested as an alternative regardless of the presence 
of any neurological symptoms. BM have a devastating impact on the patients' overall survival (OS), with a median of 6 months of survival after the manifestation of BM, and severely impair the quality of life (QoL). Presence of specific mutations in the tumor may considerably alter the course of the disease. Patients with EGFR mutations or $A L K$ rearrangements are less prone to worse prognosis. Patients presenting Diagnosis Specific Graded Prognostic Index (DS_GPA) assessment/Assessment for Lung Cancer Using Molecular Markers (Lung-mol GPA) scores 3.5-4.0 may have a median of survival of 4 years (3). This also implicates the necessity to protect the abovementioned group of patients from life threatening aspects of the disease, including BM occurrence. To do so efficiently, one has to identify the groups that may benefit the most from interventions to prevent $\mathrm{BM}$, discuss the options with the patients and consider the costs of such treatment.

\section{Prevention of BM}

Use of methods aimed at preventing BM should be considered despite the apparent lack of OS benefit as such treatment may preserve adequate neurologic and psychical aspects of QoL $(4,5)$. Higher BM rates are also associated with a greater extent of disease and as such, as evidenced by studies such as RTOG 0214 countering BMs could also be considered as means to prolong disease free survival (DFS) itself (6). It is well known that BM themselves may deteriorate neurological and physical function and thus becoming symptomatic BM. There are several options, which are to be considered as potential BM prevention tools.

\section{Chemotherapy, molecular targets, immune checkpoint inhibitors used as a prevention tool for BM occurrence}

Chemotherapy currently in routine use or used experimentally does not seem to efficiently protect patients from BM (7). The reason is the function of the bloodbrain barrier (BBB), which prohibits cytotoxic agents from reaching brain tissue by adequate concentrations. Data, so far accumulated only in non-squamous NSCLC, shows that pemetrexed used as first line therapy in advanced NSCLC may reduce the risk of developing BM as the first site of progression (8). These observations are however derived from a retrospective analysis of two randomized trials and significant results were obtained only in subgroup analyses limiting their level of evidence. Therefore, this observation has to be put into more rigorous evaluation to ascertain the real association between usage of pemetrexed and decrease the risk of $\mathrm{BM}$. There were also reports involving temozolomide usage after surgery or after platinum-based chemotherapy as a preventative measure for BM. No significant associations in terms of incidence reduction of $\mathrm{BM}$ in either of the cohorts were noted (9). However, there are promising data regarding patients with NSCLC stage III suggesting that durvalumab administered after chemoradiotherapy may decrease the incidence of BM (10). In the PACIFIC study the BM incidence was reduced 2-fold: $5.5 \%$ in the durvalumab arm vs. $11 \%$ in the control group. Durvalumab seemed to be also effective in an early analysis of a phase II trial with existing non-symptomatous, small BM, in patients with PD-L1 positive melanoma or NSCLC (11). The numbers available were, however, very low: 18 patients with NSCLC and 18 with melanoma limited the weight of this piece of evidence. Nevertheless, the responses to treatment were durable in all patients but one with NSCLC. The safety profile of this study in NSCLC patients showed grade 3 non-neurological complications such as colitis, pneumonitis fatigue or hyperkalemia to occur in some individuals alas no neurologic toxicity of grade 3 or higher was noted (11).

There are also promising initial reports of studies with osimertinib (12) and alectinib (13) aimed at preventing BM. Alectinib is a representant of modern generation of ALK inhibitors that seems to show better penetration through the BBB than the first generation of ALK inhibitors such as crizotinib, for which $\mathrm{BBB}$ concentrations equaled only $50 \%$ of blood concentrations (14). The efficacy of alectinib in terms of BM risk reduction was studied both in patients with or without previous brain irradiation $(13,15)$ and also in a comparative study with crizotinib (16). Alectinib vs. crizotinib was well studied in the Japanese population in J-ALEX study. In this trial the alectinib arm presented an advantage $v s$. crizotinib in terms of BM progression. It is of note that in this trial the proportion of patients with $\mathrm{BM}$ was higher in the crizotinib group than in the alectinib-treated one ( $28 \%$ vs. $14 \%$, respectively). In a subgroup analysis both patients with $\mathrm{BM}$ and no BM gained advantage of a prolonged progression free survival (PFS) in favor to alectinib (16). According to authors of aforementioned study —only patients without BM or with $\mathrm{BM}$ and no previous brain radiotherapy gained advantage in terms of PFS. The authors claim that the safety profile of alectinib was better than that of crizotinib, but only non- 
neurologic adverse effects were analyzed.

The efficacy of osimertinib, a third generation of ALK inhibitors, was assessed in the FLAURA trial $(12,17)$. This randomized, double blind phase III study included Asian and white population in a balanced manner and in nearly half of each arm (osimertinib vs. erlotinib or gefitinib) patients presented $\mathrm{BM}$. In the experimental arm osimertinib reduced the frequency of BM progression or death caused by BM down to $52 \%$. Safety profile was significantly in favor of osimertinib in contrast to erlotinib or gefitinib used in the control arm. Non-neurologic adverse events of grade 3 or higher occurred in $34 \%$ vs. $45 \%$ of patients, respectively. Fatal adverse events occurred in 6 patients $(2 \%)$ in the osimertinib group and in 10 patients in the standard EGFR-TKI group, but none of them were directly related to osimertinib use (12).

\section{Prophylactic cranial irradiation as a preventative tool for BM}

Given that in small-cell lung cancer (SCLC) patients prophylactic cranial irradiation (PCI) lead to the reduction of the incidence of $\mathrm{BM}$ and prolonged OS (18) further investigations were made in NSCLC patients. So far, PCI significantly reduces the incidence of $\mathrm{BM}$ and prolongs the disease-free interval in patients with NSCLC, but thus far no improvements of OS were noted $(19,20)$ even in long term observations. No randomized trials reported significant improvements of OS after PCI in patients with NSCLC. In none of the subgroups, OS prolongation was noted if PCI was used in those cohorts. This undermined the impact of PCI on the prolongation of OS regardless of patients' stage (21). The lack of impact on OS may result from a variety of causes including but not limited to the majority of patients developing extracranial metastases, low statistical power of recent studies or a true lack of biological effect. The absent effect in terms of OS prolongation may be also the result of slower accrual to studies than initially planned. It should be also taken into consideration that the treatment of BM themselves used in the control arms of studies (i.e., stereotactic radiosurgery, palliative whole brain radiotherapy, steroid intake) does seem to have a beneficial effect, showing longer than average OS times in patients not participating in such clinical trials.

Nonetheless, PCI exerts its beneficial effect by reducing the occurrence of BM by $50-70 \%$ (starting from $13 \%$ incidence in non-PCI arms with reduction by $6 \%)(21,22)$. In a recent publication of De Ruysscher et al., a meta- analysis was performed and showed that the risk ratio of developing BM when PCI is used was 0.33 (95\% CI, 0.24-0.45) (1). This effect was significant despite different radiotherapy schemes used in the trials included in the metanalysis. It is also noteworthy that the most popular radiotherapy protocols used were 30 Gy given in 10 fractions/daily 5 days a week or 30 Gy in 12 fractions in 5 days a week (see Table 1).

At least seven trials, starting from middle late 70 -ies through middle 80 s (23-26) and recent millennial trials presented a beneficial impact of $\mathrm{BM}$ in terms of reduced incidence of BM with the quality of this evidence rated as moderate by a recent systematic review (21). According to systematic reviews and several forms of bias assessment in such papers, despite nearly 40 years of performing such trials the data are still subject to a debate. In terms of that in one systematic review, some works highlight the particular risk of conducting bias (28). In those reviewers' opinion, however, it was finally assessed as low risk of introducing bias. The latest studies showed that the incidence of BM was in fact higher than expected in both arms, probably as a consequence of better diagnostic tools. In studies performed in late $70 \mathrm{~s}-80 \mathrm{~s}$ only limited image brain diagnostics were available and were not always performed prior to study enrolment. In studies, which have started in the new millennium, the inclusion criteria involved baseline brain diagnostics performed with at least a CT with contrast evaluation but preferably using high-quality MRI. Table 1 contains an outline of the main studies which included PCI as part of treatment of NSCLC. Trials from the $\mathrm{XX}^{\text {th }}$ century (apart from SWOG study) recruited patients with diseases of stage I to III. In recent randomized multicenter studies (NVALT11/DLCRG-02 or RTOG 0214, Li et al.), including those with long-term observations, the focus is on patients with locally advanced diseases (stage IIIA-IIIB-AJCC $7^{\text {th }}$ edition), who are expected to be at a greater risk of developing BM. In the above listed studies $\mathrm{BM}$ were considered as a primary endpoint or secondary one after OS or DFS. In the NVALT11/DLCRG-02 study no significant differences were shown in terms of DFS at 2 years: $67 \%$ in PCI arm vs. $72 \%$ in the observation arm (1). On the other hand, the long-term observation of in RTOG 0214 showed a significantly prolonged DFS in patients receiving PCI (6). In the $\mathrm{Li}$ et al. study, where patients of Asian ethnicity were recruited, the DFS was used as a primary endpoint and was significantly longer in the PCI than in the control arms showing a difference of median survival times of 28.5 vs. 21.2 months (27). An 


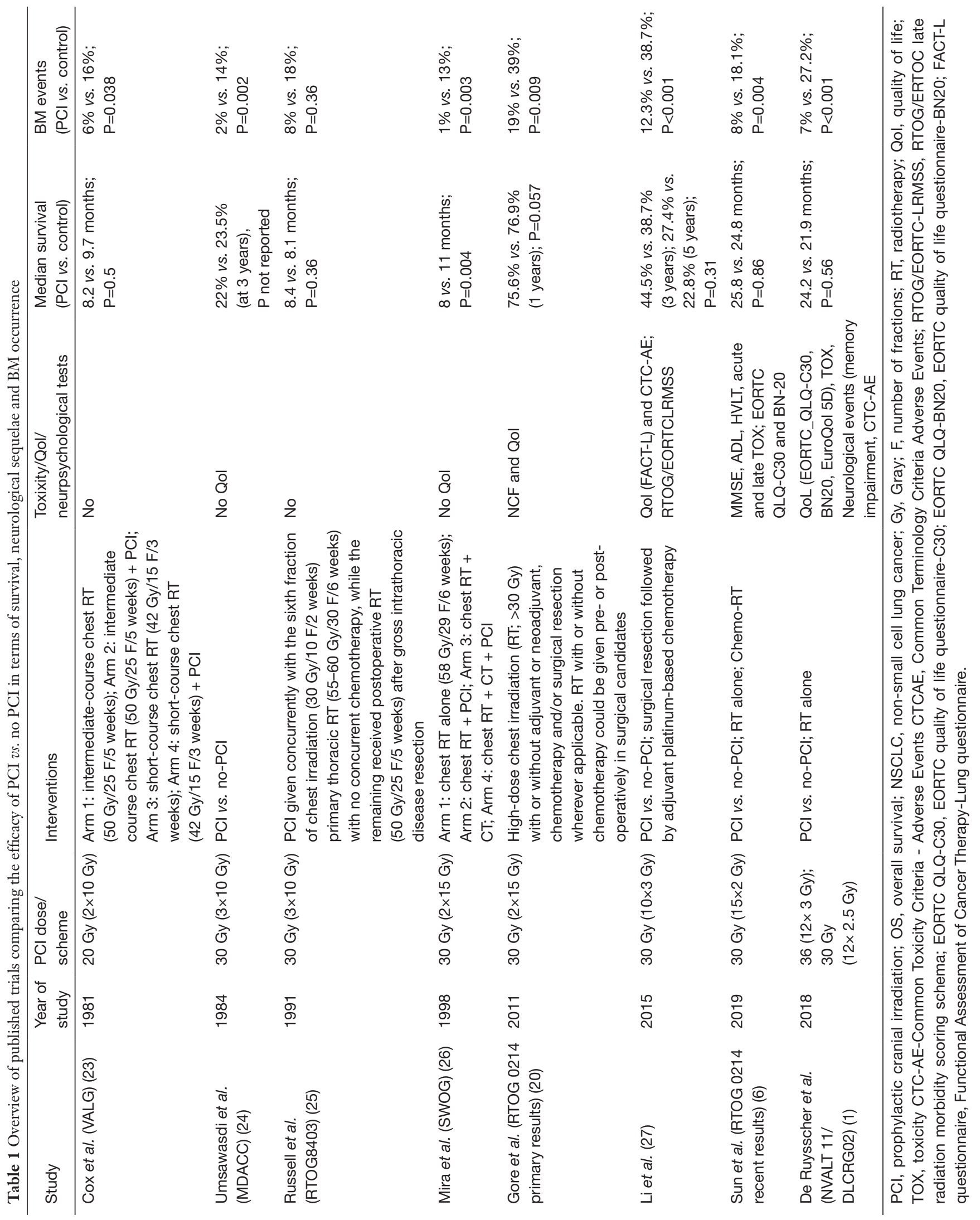


important aspect of this study was that all patients analyzed in that paper had undergone surgery-which may have been a favorable factor pro allowing the authors to obtain adequately long time of observation.

In all recent studies the incidence of $\mathrm{BM}$ was shown to be significantly lower in PCI than in respective control arms. NVALT11/DLCRG-02 study addressed the occurrence of symptomatic BM as an endpoint. This was defined as BM identified during control MRI and the patient manifesting at least one of the following symptoms: vomiting, cognitive or affective disturbances, seizures, and focal neurologic symptoms. The rationale behind this was to show how PCI can be effective in preventing symptomatic $\mathrm{BM}$ in this specific cohort and thus preventing QoL deterioration (1). In the NVALT11/DLCRG-02 study $7 \%$ (PCI group) vs. 24.2\% (controls) patients developed symptomatic $\mathrm{BM}$ yielding an absolute reduction of nearly $20 \%$ probability over time (1). In the RTOG 0214 there was no mention about whether BM being symptomatic or not, however, the 10 -year $\mathrm{BM}$ rates equaled $16.7 \%$ in the PCI arm and $28.3 \%$ in the observation arm making it highly likely that the proportion of symptomatic BM was reduced in a proportional manner (6). Li et al. did not specify any subgroup of BM with symptoms either, but again, the 5 -year brain recurrence rate was $15.6 \%$ in the PCI arm vs. $45.3 \%$ in no-PCI arm (29). Should this proportion be maintained for symptomatic BM reduction, PCI may have a marked impact on preventing the QoL decline due to BMassociated symptoms.

Patient status (WHO) or previous treatment (chemotherapy, surgery) had no impact in the NVALT11/ DLCRG-02 study on the occurrence of BM in planned subgroup analysis (1). A different study performed by Sun et al., also in a planned subgroup analysis, reported that younger patients ( $<60$ years old) and those with nonsquamous disease develop BM more often than older patients and squamous histology (6). In the work by Li et al. such analyses were not performed or reported (29).

In both NVALT11/DLCRG-02 and RTOG 0214 trials, all patients had undergone surgery, radiotherapy and chemotherapy which were a standard of care at time of patients' accrual $(1,6)$. In the trial by Li et al. it was stated that no postoperative radiation therapy was performed in patients in whom N2 status was revealed in the pathology report (27).

None of those three studies reported molecular data such as the mutation status of the treated tumors. Similarly, there are no information about subsequent treatment of BM in the control arms of any of the abovementioned recent studies. All of those trials, as well as previous ones suffered from very low accrual.

Despite the substantial reduction of the risk of developing BM, and data suggesting a DFS prolongation as well, PCI is not free of its own risks. The major limiting factor is that PCI may lead to radiation-induced toxicity. Therefore, complication of PCI and BM may both exert a similarly negative impact on cognitive function. The specific types of neurocognitive deterioration associated with $\mathrm{BM}$ occurrence depend on their location and damage incurred to nearby regions of the brain. PCI on the other hand induces a specific type of deterioration, which manifests its impact typically 3 months after the procedure in the form of mild deterioration of cognitive functions.

Toxicity assessed by a physician is more and more often taken into consideration as an important secondary endpoint or being a point of interest of separate publications $(30,31)$. Typically, neurologic but also non-neurologic symptoms are assessed in patients who underwent PCI. Neurologic and non-neurologic events which may develop after PCI and associated toxicity include typically mild cognitive decline and mild headaches. However, the fear of a very serious or life-threating neurologic events is very prevalent and treatment-limiting. It is critical to note that the tests used to assess neurologic functions are not very specific and are one typically used for dementia screening. This is associated with limitations arising from the lack of validation of studies in other cohorts of patients with cognitive impairment. The most commonly used tools are mini mental state examination (MMSE) (32), Hopkins Verbal Learning Test (HVLT) (33) or Activities of Daily Living (ADL) (34).

Non-neurologic toxicity of PCI predominantly manifests as vomiting, which intensity dwindles with time, while the long-lasting neurologic toxicities exacerbate as time from treatment elapses. The non-neurologic sequelae of PCI are mainly assessed using the EORTC QLQ-C30 and brainspecific EORTC questionnaires (QLQ-BN20).

Evolution of adverse events overtime was a subject of the NVALT11/DLCRG-02 study. In the PCI arm of NVALT11/DLCRG-02 neurologic adverse events tended to develop overtime but were mild (grade 1 to 2 ). The worsening of QoL scores for the patients occurred during the time of treatment or soon after the treatment, mainly due to the appearance of immediate non-neurologic symptoms (1).

In the RTOG 0214 study over a 10-year follow up in terms of neurocognitive function the authors claimed that 
there were insufficient data to conclude anything about long term neurocognitive toxicity (6).

An earlier pooled analysis of the RTOG 0214 data was performed on the basis of neurological evaluation with MMSE and HVLT. The results did not reveal any signs of a serious mental decline 1 year after PCI. Pooled analysis of RTOG 0214 and RTOG 0212 revealed that the deterioration of QoL may be considered as a good surrogate for poor OS (18).

$\mathrm{Li}$ et al. reported similar results: the main acute toxicities of a grade of at least 3 , associated with PCI occurred within 90 days of treatment start. The reported complications included headache (in $1 \%$ of patients) and fatigue (2\%). Severe headaches after 90 days were noted in $25 \%$ of the patients, fatigue in $1 \%$ and severe skin atrophy was observed in 1 individual. No significant differences between the two arms were shown in terms of QoL questionnaires.

The risk of neurologic symptoms (especially neurocognitive function decline) caused by PCI, may be potentially reduced by hippocampal avoidance (HA) (35) or the use of memantine (36). Preventing mental deterioration by using whole brain radiotherapy (WBRT)-as a standard technique used for PCI - plus memantine with or without HA showed, in a phase III trial, that patients with HA present no increased risk of worse control and HA protects from neurocognitive deterioration (36). Memantine plus HA was associated with lower risk of deterioration in contrast to control arm (HR $=0.74, \mathrm{P}=0.02)$ (36).

The NVALT11/DLCRG-02 study emphasized also patient-reported occurrence of adverse events. The numbers reported by patients in terms of vomiting, dizziness, headache, hypersomnia, memory impairment were in fact higher in PCI arm than in the observational arm (55 of 87 patients $v$ s. 36 of 88 patients, correspondingly) but the grades of such outcomes was assessed as mild (1).

Given that the tests used for evaluation of neurologic deterioration performed in recent studies involving PCI are mostly adapted screening tests for cognitive impairment of any kind. This creates a major need for the wider use of other, more sensitive tools that should be used to assess mild neurologic deterioration or an effect of prophylaxis using memantine or HA in PCI. Such tests do exist but are still not validated in cohorts of patients with cancer. One such tool, designed for the assessment of mild memory and cognitive function impairment is a 10-minute cognitive screening tool-the Montreal Cognitive Assessment (MoCA) (37). This test is designed to be used as a high-sensitivity tool that would identify impairment mild enough to slip through the MMSE test, with an intent for the MoCA to be used by primary care physicians. MoCA was already validated not only in US, Brazilian, and Asian populations with dementia but also on patients with stroke and in terms of neurological and cognitive rehabilitation effects. Another promising questionnaire is the Brain Health Assessment (BHA). This validated 10-minute, tablet-based test assesses aspects of neural performance other than memory (executive functions, speed, visuospatial skills or language), as these areas may deteriorate before overt memory failure develops (38). Given the improvements in survival rates and the pressure to preserve QoL in cancer survivors it becomes more and more reasonable to perform baseline cognitive performance assessment before initiating treatment (with or without PCI) to better monitor the patients. The abovementioned tools, or close collaboration with clinical psychologists or psychiatrists, will become more and more important in safeguarding patients from cognitive and neurological complications.

\section{Cost-effectiveness of preventing BM (PCI and other forms of preventing BM)}

BM in patients diagnosed with NSCLC imposes a very high economic burden. It is therefore of utmost importance to analyse and compare different methods of BM prevention or treatment not only in terms of efficacy but also in terms of healthcare costs and their derivates. These may include outpatient and inpatient costs together with pharmacy costs, work absenteeism and potential dependence on social care. Those measures should be standardized (i.e., by average monthly expenditure per patient) and ideally should be adjusted for inflation in specific countries. Those kinds of analyses bring also a true light in real cost load of BM prevention and treatment. Cost-effectiveness analyses typically rely on cost utility analyses, where quality adjusted life years (QUALY) are primary outcomes. Those analyses should be standardized by appropriate reporting (39). According to American Society of Clinical Oncology (ASCO), there is currently highlighting on interventions demonstrating value instead of just effectiveness, which is of utmost importance for health care payer.

Thus far, analyses of cost reduction were performed mostly for molecularly targeted therapies and usually are performed on datasets obtained during the execution of clinical trials. This was the case for the analysis performed on the cohort assembled during the ALEX study $(40,41)$. 
The cost associated with $\mathrm{BM}$ treatment in abovementioned study was estimated as $6,029 \$$ per month over a $24-$ month period of total treatment. When the alectinib and crizotinib-treated groups were compared, it was shown that alectinib reduced those costs by approximately $41,000 \$$ per patient simply by reducing the incidence of BM. However, the analysis did not include the costs of additional alectinib treatment, and did not consider the additional expenditure associated with using alectinib instead of crizotinib (40).

So far, the cost-effectiveness of analysis or estimation of costs of PCI was not assessed in patients with NSCLC. De Ruysscher $e t$ al. claimed to evaluate the health costs but their analysis focused on the estimation of costs as associated with adverse events outcomes, as these are an integral component of such analyses, but no cost-effectiveness analyses were published from this cohort thus far (1). Cost-effectiveness analysis of PCI was performed in SCLC patients by means of a Markov model with internal validation, where PCI with HA (Arc therapy) was used. This treatment modality seemed to be more effective than conventional PCI assuming the risk of developing $\mathrm{BM}$ on level of $14 \%$ or by preventing neurocognitive dysfunction by at least $40 \%$ rates reduction (42). The two-way sensitivity analysis performed in this study revealed that PCI with HA would be the preferred strategy if every $10 \%$ decrease of neurocognitive disfunction brings less expenses than increased risk of new metastases to $3 \%$ (42).

\section{Future development of $\mathrm{PCl}-$ current ongoing studies}

There are several recently started, ongoing trials registered on clinicaltrials.gov:

(I) Prophylactic Cranial irradiation in erlotinib/ gefitinib-responders with NSCLC-RT1001trial identifier NCT01158170 (6) - this phase III trial, taking place in China, includes patients with NSCLC stage III after response to erlotinib/gefitinib treatment. The primary endpoint is BM incidence, secondary endpoints are OS, safety profile and tolerability, QoL. Status-unknown.

(II) Hippocampal-sparing prophylactic cranial irradiation in pathologically nodal positive non-small cell lung cancer-NCT02448992 (43). This phase II/III study will assess PCI with hippocampal spring $v s$. no PCI in patients with NSCLC stage III N+after completion of chemotherapy. This study has already stopped recruitment. Its estimated completion date is December 2020. The primary endpoint is $\mathrm{BM}$ incidence, irrespective of symptoms, secondary endpoints are the effects of hippocampal-sparing $\mathrm{PCI}$ on neurocognitive functions (NCF).

\section{Conclusions}

PCI may be of use as a tool of preventing BM, especially symptomatic ones, but it demands a very careful analysis of pros and cons with the patient. The impact of PCI on OS in NSCLC, even in highly selective groups of patients or with the use of neuroprotective techniques or supportive care will be marginal if any at all. However, the impact may be highly beneficial in terms of improved QoL if toxicity is avoided. Given the need for an individualized approach to PCI use, there is an urgent need to identify and define the group that has the greatest potential benefit from any kind of preventing BM. Without such guidelines it will be hard to optimize the use of PCI in NSCLC in an evidencedriven way. The effect of PCI in specific subgroups of patients: younger individuals with adenocarcinomas, staged III disease, patients with a high volume of disease, individuals with tumors with ALK translocations or EGFR mutations is particularly interesting as these individuals may benefit from group-specific effects of PCI and other preventative measures against $\mathrm{BM}$. The use of protection or sparring brain from toxicity is also of interestmainly through the use of memantine and HA (or at least administering radiation dose as low as reasonably acceptable in this organ at risk) by means of new techniques of radiotherapy. By minimizing toxicity one can expect a reduction of $\mathrm{BM}$ risk, with a potential benefit in terms of QoL and prolongation of DFS, or perhaps even OS in long term observations. Therefore, optimized and tailored reduction of BM risk seems to be a highly costeffective treatment. Unfortunately, performing studies aimed at the optimization of PCI and toxicity prevention might be very difficult due to limited recruitment performance. Therefore, the most urgent need is to devise optimal strategies for recruiting individuals to BMprevention trials and until high quality data are obtained, individualize the treatment approach when deciding upon PCI use in patients with cancer.

\section{Acknowledgments}

I wish to acknowledge the help provided by Wojciech Fendler. M.D. Ph.D. in writing and language editing 
assistance. This article was not financially supported. Funding: None.

\section{Footnote}

Provenance and Peer Review: This article was commissioned by the Guest Editor (Lucyna Kepka) for the series "Radiotherapy for Brain Metastases from Lung Cancer" published in Fournal of Thoracic Disease. The article has undergone external peer review.

Conflicts of Interest: All authors have completed the ICMJE uniform disclosure form (available at http://dx.doi. org/10.21037/jtd.2019.11.36). The series "Radiotherapy for Brain Metastases from Lung Cancer" was commissioned by the editorial office without any funding or sponsorship. LK served as the unpaid Guest Editor of the series and serves as an unpaid editorial board member of fournal of Thoracic Disease from Aug 2018 to Jul 2020. The other author has no other conflicts of interest to declare.

Etbical Statement: The authors are accountable for all aspects of the work in ensuring that questions related to the accuracy or integrity of any part of the work are appropriately investigated and resolved.

Open Access Statement: This is an Open Access article distributed in accordance with the Creative Commons Attribution-NonCommercial-NoDerivs 4.0 International License (CC BY-NC-ND 4.0), which permits the noncommercial replication and distribution of the article with the strict proviso that no changes or edits are made and the original work is properly cited (including links to both the formal publication through the relevant DOI and the license). See: https://creativecommons.org/licenses/by-nc-nd/4.0/.

\section{References}

1. De Ruysscher D, Dingemans AMC, Praag J, et al. Prophylactic cranial irradiation versus observation in radically treated stage III non-small-cell lung cancer: A randomized phase III NVALT-11/DLCRG-02 study. J Clin Oncol 2018;36:2366-77.

2. NCCN. Small cell lung cancer Small cell lung cancer [Internet]. Vol. 7, NCCN. 2007. p. 1-8. Available online: https://www.nccn.org/professionals/physician_gls/pdf/ nscl.pdf

3. Sperduto PW, Yang TJ, Beal K, et al. Estimating survival in patients with lung cancer and brain metastases an update of the graded prognostic assessment for lung cancer using molecular markers (Lung-molGPA). JAMA Oncol 2017;3:827-31.

4. Verhaak E, Gehring K, Hanssens PEJ, et al. Health-related quality of life of patients with brain metastases selected for stereotactic radiosurgery. J Neurooncol 2019;143:537-46.

5. Fenske DC, Price GL, Hess LM, et al. Systematic Review of Brain Metastases in Patients With Non-Small-Cell Lung Cancer in the United States, European Union, and Japan. Clin Lung Cancer 2017;18:607-14.

6. Sun A, Hu C, Wong SJ, et al. Prophylactic Cranial Irradiation vs Observation in Patients with Locally Advanced Non-Small Cell Lung Cancer: A Long-term Update of the NRG Oncology/RTOG 0214 Phase 3 Randomized Clinical Trial. JAMA Oncol 2019;5:847-55.

7. Tsakonas G, De Petris L, Ekman S. Management of brain metastasized non-small cell lung cancer (NSCLC) - From local treatment to new systemic therapies. Cancer Treat Rev 2017;54:122-31.

8. Ortuzar W, Hanna N, Pennella E, et al. Brain metastases as the primary site of relapse in two randomized phase III pemetrexed trials in advanced nonsmall-cell lung cancer. Clin Lung Cancer 2012;13:24-30.

9. Boggs DH, Robins HI, Langer CJ, et al. Strategies to prevent brain metastasis in high-risk non-small-cell lung cancer: Lessons learned from a randomized study of maintenance temozolomide versus observation. Clin Lung Cancer 2014;15:433-40.

10. Antonia SJ, Villegas A, Daniel D, et al. Durvalumab after chemoradiotherapy in stage III non-small-cell lung cancer. N Engl J Med 2017;377:1919-29.

11. Goldberg SB, Gettinger SN, Mahajan A, et al. Pembrolizumab for patients with melanoma or non-smallcell lung cancer and untreated brain metastases: early analysis of a non-randomised, open-label, phase 2 trial. Lancet Oncol 2016;17:976-83.

12. Soria JC, Ohe Y, Vansteenkiste J, et al. Osimertinib in untreated EGFR-Mutated advanced non-small-cell lung cancer. N Engl J Med 2018;378:113-25.

13. Zhang I, Zaorsky NG, Palmer JD, et al. Targeting brain metastases in ALK-rearranged non-small-cell lung cancer. Lancet Oncol 2015;16:e510-21.

14. Togashi Y, Masago K, Masuda S, et al. Cerebrospinal fluid concentration of gefitinib and erlotinib in patients with non-small cell lung cancer. Cancer Chemother Pharmacol 2012;70:399-405.

15. Camidge DR, Dziadziuszko R, Peters S, et al. Updated 
Efficacy and Safety Data and Impact of the EML4-ALK Fusion Variant on the Efficacy of Alectinib in Untreated ALK-Positive Advanced Non-Small Cell Lung Cancer in the Global Phase III ALEX Study. J Thorac Oncol 2019;14:1233-43.

16. Hida $T$, Nokihara $H$, Kondo $M$, et al. Alectinib versus crizotinib in patients with ALK-positive non-small-cell lung cancer (J-ALEX): an open-label, randomised phase 3 trial. Lancet 2017;390:29-39.

17. Ramalingam SS, Rukazenkov Y, Thomas K, et al. A randomized, phase III study (FLAURA) of AZD9291, a novel EGFR-TKI, versus gefitinib or erlotinib in treatment-naïve patients with advanced non-small cell lung cancer and an EGFR-TKI-sensitizing mutation. J Clin Oncol 2015;33:abstr TPS8102.

18. Redmond KJ, Hales RK, Anderson-Keightly H, et al. Prospective Study of Hippocampal-Sparing Prophylactic Cranial Irradiation in Limited-Stage Small Cell Lung Cancer. Int J Radiat Oncol Biol Phys 2017;98:603-11.

19. Movsas B, Bae K, Meyers C, et al. Phase III Study of Prophylactic Cranial Irradiation vs. Observation in Patients with Stage III Non-small-cell Lung Cancer: Neurocognitive and Quality of Life Analysis of RTOG 0214. Int J Radiat Oncol 2009;75:S1.

20. Gore EM, Paulus R, Wong S, et al. Phase III Comparison of Prophylactic Cranial Irradiation Versus Observation in Patients with Locally Advanced Non-small Cell Lung Cancer -- An Updated Analysis of RTOG 0214. Int J Radiat Oncol 2012;84:S103.

21. Al Feghali KA, Ballout RA, Khamis AM, et al. Prophylactic cranial irradiation in patients with non-smallcell lung cancer: A systematic review and meta-analysis of randomized controlled trials. Front Oncol 2018;8:115.

22. Krawczyk P, Duchnowska R, Nicoś M, et al. Preventing central nervous system metastases in non-small cell lung cancer. Expert Rev Anticancer Ther 2018;18:1077-83.

23. Cox JD, Stanley K, Petrovich Z, et al. Cranial irradiation in cancer of the lung of all cell types. JAMA 1981;245:469-72.

24. Umsawasdi T, Valdivieso M, Chen TT, et al. Role of elective brain irradiation during combined chemoradiotherapy for limited disease non-small cell lung cancer. J Neurooncol 1984;2:253-9.

25. Russell AH, Pajak TE, Selim HM, et al. Prophylactic cranial irradiation for lung cancer patients at high risk for development of cerebral metastasis: results of a prospective randomized trial conducted by the Radiation Therapy Oncology Group. Int J Radiat Oncol Biol Phys
1991;21:637-43.

26. Mira JG, Miller TP, Crowley JJ. Chest irradiation (RT) vs. chest RT + chemotherapy \pm prophylactic brain RT in localized non small cell lung cancer: A southwest oncology group randomized study. Int J Radiat Oncol Biol Phys 1990;19:145.

27. Li N, Zeng ZF, Wang SY, et al. Randomized phase III trial of prophylactic cranial irradiation versus observation in patients with fully resected stage IIIA-N2 nonsmallcell lung cancer and high risk of cerebral metastases after adjuvant chemotherapy. Ann Oncol 2015;26:504-9.

28. Witlox WJA, Ramaekers BLT, Zindler JD, et al. The Prevention of Brain Metastases in Non-Small Cell Lung Cancer by Prophylactic Cranial Irradiation. Front Oncol 2018;8:241.

29. Aupérin A, Arriagada R, Pignon JP, et al. Prophylactic cranial irradiation for patients with small-cell lung cancer in complete remission. N Engl J Med 1999;341:476-84.

30. Gondi V, Paulus R, Bruner D, et al. Prognostic significance of QOL deterioration during early lung cancer survivorship: Secondary analysis of RTOG 0212 and 0214. J Clin Oncol 2011;29:6061.

31. Sun A, Bae K, Gore EM, et al. Phase III trial of prophylactic cranial irradiation compared with observation in patients with locally advanced non-small-cell lung cancer: Neurocognitive and quality-of-life analysis. J Clin Oncol 2011;29:279-86.

32. Ciesielska N, Sokołowski R, Mazur E, et al. Czy test Montreal Cognitive Assessment (MoCA) moze być skuteczniejszy od powszechnie stosowanego Mini-Mental State Examination (MMSE) w wykrywaniu łagodnych zaburzeń funkcji poznawczych u osób po 60. roku zycia? Metaanaliza. Psychiatr Pol 2016;50:1039-52.

33. Sawyer RJ, Testa SM, Dux M. Embedded performance validity tests within the Hopkins Verbal Learning TestRevised and the Brief Visuospatial Memory Test-Revised. Clin Neuropsychol 2017;31:207-18.

34. Luttenberger K, Reppermund S, Schmiedeberg-Sohn A, et al. Validation of the Erlangen Test of Activities of Daily Living in Persons with Mild Dementia or Mild Cognitive Impairment (ETAM). BMC Geriatr 2016;16:111.

35. Belderbos J, van Kesteren Z, De Ruysscher D, et al. Hippocampus Avoiding Prophylactic Brain Irradiation for NSCLC Patients: A Simple Technique. Int J Radiat Oncol 2011;81:S615-6.

36. Tomé W, Deshmukh S, Gondi V, et al. Radi-11. Nrg Oncology Cc001: a Phase Iii Trial of Hippocampal Avoidance in Addition To Whole-Brain Radiotherapy 
(Wbrt) Plus Memantine To Preserve Neurocognitive Function in Patients With Brain Metastases (Bm). NeuroOncology Adv 2019;1:i23-4.

37. Bourgeois-Marcotte J, Flamand-Roze C, Denier C, et al. LAST-Q: adaptation et normalisation franco-québécoises du Language Screening Test. Rev Neurol (Paris) 2015;171:433-6.

38. Possin KL, Moskowitz T, Erlhoff SJ, et al. The Brain Health Assessment for Detecting and Diagnosing Neurocognitive Disorders. J Am Geriatr Soc 2018;66:150-6.

39. Husereau D, Drummond M, Petrou S, et al. Consolidated Health Economic Evaluation Reporting Standards (CHEERS) statement. Eur J Health Econ 2013;14:367-72. 40. Burudpakdee C, Wong W, Seetasith A, et al. Economic

Cite this article as: Chalubinska-Fendler J, Kepka L. Prophylactic cranial irradiation in non-small cell lung cancer: evidence and future development. J Thorac Dis 2021;13(5):32793288. doi: $10.21037 /$ jtd.2019.11.36 impact of preventing brain metastases with alectinib in ALK-positive non-small cell lung cancer. Lung Cancer 2018;119:103-11.

41. Shaw AT, Peters S, Mok T, et al. Alectinib versus crizotinib in treatment-naive advanced ALK -positive non-small cell lung cancer (NSCLC): Primary results of the global phase III ALEX study. J Clin Oncol 2017;35:abstr LBA9008.

42. Qu XM, Mishra M V., Bauman GS, et al. Costeffectiveness of prophylactic cranial irradiation with hippocampal avoidance in limited stage small cell lung cancer. Radiother Oncol 2017;122:411-5.

43. NCT02448992. Available online: https://clinicaltrials.gov/ ct $2 /$ results? cond $=\&$ term $=02448992+\&$ cntry $=\&$ state $=\&$ city $=\&$ dist $=$ 